\title{
A NOTE ON UNIFORM CONVERGENCE OF STOCHASTIC PROCESSES
}

\author{
JOHN B. WALSH
}

Let $T$ be a compact metrizable space and let $\left\{X_{n}(t), t \in T\right\}$ $n=1,2, \cdots$ be independent continuous (w.p.1.) stochastic processes with mean zero. We apply an abstract martingale convergence theorem to show that if $\sum X_{n}(t)$ converges pointwise with probability one to a continuous stochastic process with an integrable norm, it also converges uniformly in $t$ with probability one. Among the immediate consequences of this theorem is a generalization of Paley and Wiener's result on the uniform convergence of the Fourier expansion of Brownian motion.

1. Let $\mathfrak{X}$ be a Banach space with norm \|\| , and let $(\Omega, \mathfrak{F}, P)$ be a probability space. If $X$ is a strong random variable with values in $\mathfrak{X}$ such that $E\{\|X\|\}<\infty$, and if $\mathfrak{F}_{1} \subset \mathfrak{F}$ is a Borel field, we will denote the strong conditional expectation of $X$ given $\mathfrak{F}_{1}$ by $E\left\{X \mid \mathfrak{F}_{1}\right\}$. (For definitions and properties of strong random variables and strong conditional expectations, see [6].)

The following theorem is due to Chatterji [1, Theorems 1 and 4].

Theorem A (ChATTERJI). Let $X$ be a strong random variable with values in $\mathfrak{X}$ and let $\left\{X_{n}, \mathfrak{F}_{n}, n \geqq 1\right\}$ be a martingale, where $X_{n}=E\left\{X \mid \mathfrak{F}_{n}\right\}$, $n=1,2, \cdots$. If for some $p, 1 \leqq p<\infty, E\{\|X\| p\}<\infty$, then there is a strong random variable $Y$ such that

(A1) $\left\|X_{n}-Y\right\| \rightarrow 0$ w.p.1.

(A2) $E\left\{\left\|X_{n}-Y\right\|^{p}\right\} \rightarrow 0$

and $Y=E\left\{X \mid \mathfrak{F}_{\infty}\right\}$ where $\mathfrak{F}_{\infty}$ is the Borel field generated by $\cup \mathfrak{F}_{n}$.

2. In what follows, $T$ is compact and metrizable and $C(T)$ is the Banach space of continuous functions on $T$ with the sup-norm. If $\{X(t), t \in T\}$ is a continuous stochastic process on $(\Omega, \mathfrak{F}, P)$, it determines a random variable $X$ with values in $C(T)$. It is a straightforward consequence of the separability of $T$ and $C(T)$ coupled with the measurability of $X(t)$ for each $t$ that $X$ is a strong random variable.

3. Let $\{X(t), t \in T\}$ be a continuous stochastic process satisfying $E\left\{\sup _{t \in T}|X(t)|\right\}<\infty$ and let $\left\{X_{n}(t), t \in T\right\} \quad n=1,2, \cdots$ be a sequence of independent continuous stochastic processes with mean

Received by the editors April 14, 1966. 
zero. Let $S_{n}(t)=\sum_{1}^{n} X_{j}(t)$ and let $\mu$ be a finite regular Borel measure on $T$ whose smallest closed support is $T$ itself. Then we have

THEOREM B. The following are equivalent:

(B1) $S_{n}(t) \rightarrow X(t)$ uniformly in $t$ w.p.1.

(B2) For some strictly monotone increasing function $\Phi$ on $[0, \infty)$, continuous at zero, with $\Phi(0)=0$ :

$$
\int_{T} \Phi\left(\left|S_{n}(t)-X(t)\right|\right) d \mu(t) \rightarrow 0 \quad \text { w.p.1. }
$$

(B3) For each $t$ in some countable dense subset $T_{0} \subset T, S_{n}(t) \rightarrow X(t)$ w.p.1.

Proof. (B1) $\Rightarrow(B 2)$ is obvious for any such $\Phi$. (B2) $\Rightarrow$ (B3) follows from an application of Fubini's theorem and the fact that a sum of independent random variables which converges in measure also converges w.p.1. The only nontrivial part is $(\mathrm{B} 3) \Rightarrow(\mathrm{B} 1)$.

For each $n$, let $\mathfrak{F}_{n}$ be the Borel field generated by $\left\{X_{1}(t), \cdots, X_{n}(t)\right.$, $t \in T\}$ and consider

$$
E\left\{X(t)-S_{n}(t) \mid \mathfrak{F}_{n}\right\} \quad \text { for } t \in T_{0} .
$$

$X(t)$ is absolutely integrable since $\sup _{t \in T}|X(t)|$ is, and by (B3) $X(t)$ is the a.e. limit of a sum of independent mean zero random variables, hence $E\{X(t)\}=0[3$, Theorem 5.1, p. 373].

Further, $X(t)-S_{n}(t)=\sum_{n+1}^{\infty} X_{j}(t)$, which is independent of $\mathfrak{F}_{n}$ so that

$$
E\left\{X(t)-S_{n}(t) \mid \mho_{\mho_{n}}\right\}=E\left\{X(t)-S_{n}(t)\right\}=0 ;
$$

or, since $S_{n}(t)$ is measurable $\mathfrak{F}_{n}$,

$$
E\left\{X(t) \mid \mathfrak{\Im}_{n}\right\}=S_{n}(t) .
$$

Let $x_{t}^{\prime}$ be the evaluational functional on $C(T)$, i.e. if $\xi \in C(T)$, $x_{i}^{\prime}(\xi)=\xi(t)$. Let $X$ and $S_{n}$ be the random variables with values in $C(T)$ determined by $\{X(t), t \in T\}$ and $\left\{S_{n}(t), t \in T\right\}$ respectively. $E\{\|X\|\}$ $<\infty$ by hypothesis so that $E\left\{X \mid \mathfrak{F}_{n}\right\}$ exists and we have for $t \in T_{0}$ :

$$
x_{t}^{\prime}\left(E\left\{X \mid \mathfrak{F}_{n}\right\}-S_{n}\right)=E\left\{x_{t}^{\prime}(X) \mid \mathfrak{F}_{n}\right\}-S_{n}(t)=0 \quad \text { w.p.1. }
$$

where we have used the fact that a continuous linear functional commutes with the strong conditional expectation operator $[6$, p. 357]. $T_{0}$ is countable so (3.2) holds w.p.1. simultaneously for all $t \in T_{0} . T_{0}$ is dense in $T$, so the set $\left(x_{t}^{\prime}, t \in T_{0}\right)$ separates points of $C(T)$, hence

$$
E\left\{X \mid \mathfrak{F}_{n}\right\}=S_{n} \quad \text { w.p.1. }
$$


Therefore by Theorem A, the sequence $\left(S_{n}\right)_{n \in N}$ converges strongly w.p.1. The only possible limit is $X$, so $S_{n}(t) \rightarrow X(t)$ uniformly in $t$ w.p.1.

Note. Let $y_{1}, y_{2}, \ldots$ be independent mean zero random variables and let $S_{n}=\sum_{1}^{n} y_{n}$. If for some subsequence $\left(n_{k}\right),\left(S_{n_{k}}\right)$ converges w.p.1. to an integrable random variable $x$, the whole sequence converges to $x$. Using this remark, we see that we can replace the convergence in any or all of (B1), (B2), and (B3) with convergence along some subsequence and the theorem still holds.

If we apply the mean convergence part of Theorem A we get

Corollary 1. If $E\{\sup |X(t)| p: t \in T\}<\infty$ for some $p, 1 \leqq p<\infty$, then any of (B1), (B2) or (B3) implies

$$
E\left\{\sup \left|X(t)-S_{n}(t)\right|^{p}: t \in T\right\} \rightarrow 0 \text { as } n \rightarrow \infty .
$$

4. Applications-Brownian motion. Let $\eta_{1}, \eta_{2}, \cdots$ be a sequence of independent normal random variables with mean zero and variance one. Let $a>0$ and let $\phi_{1}, \phi_{2}, \ldots$ be any complete orthonormal sequence in $L^{2}[0, a]$. Define

$$
\Phi_{j}(t)=\int_{0}^{t} \phi_{j}(u) d u \quad j=1,2, \cdots
$$

L. A. Shepp [7] has proved that

(4.1) for each $t$ the series $\sum_{1}^{\infty} \eta_{j} \Phi_{j}(t)$ converges w.p.1. to $Z(t)$, where $\{Z(t): 0 \leqq t \leqq a\}$ is separable Brownian motion from the origin, and McKean has shown that

(4.2) the convergence in (4.1) is even uniform in $t$ w.p.1. We have an immediate independent proof of (4.2) since $Z(t)$ is continuous w.p.1. [3, p. 393], and by the reflection principle [3, p. 392], $\sup _{0 \leqq t \leqq a}|Z(t)|{ }^{p}$ is in tegrable for any $p>0$. For each $j,\left\{\eta_{j} \phi_{j}(t), 0 \leqq t \leqq a\right\}$ is a continuous process with mean zero and by (4.1) the partial sums converge for each $t$ w.p.1. so by Theorem B and Corollary 1, we get:

CoRollaRy 2. $\sum \eta_{j} \Phi_{j}(t)$ converges uniformly in $t$ w.p.1., and moreover $E\left\{\sup _{0 \leqq t \leqq a}\left|Z(t)-\sum_{1}^{n} \eta_{j} \Phi_{j}\right| p\right\} \rightarrow 0$ as $n \rightarrow \infty$ for all $p>0$.

This includes as a special case the result of Paley and Wiener [5]: If $\eta_{0}, \eta_{1}, \cdots$ and $\eta_{1}^{\prime}, \eta_{2}^{\prime}, \cdots$ are independent normal mean zero and variance one random variables, then

$$
\eta_{0} t+\frac{1}{2 \pi} \sum_{1}^{\infty} \frac{1-\cos 2 \pi n t}{n} \eta_{n}+\frac{1}{2 \pi} \sum_{1}^{\infty} \frac{\sin 2 \pi n t}{n} \eta_{n}{ }^{\prime}
$$

converges uniformly to $Z(t)$ in $[0,1]$. Paley and Wiener actually 
proved only that a subsequence of partial sums converges, whereas we see that the whole sequence converges, a fact also proved by Delporte [2].

5. Eigenfunction expansions. Let $\{X(t), t \in[0, a]\}$ be a continuous gaussian process with mean zero and covariance function $\Gamma(s, t)$, $x, t \in[0, a]$. Consider the normalized eigenfunctions $\phi_{k}$ and eigenvalues $\lambda_{k}$ of the equation

$$
\lambda \phi(s)=\int_{0}^{a} \Gamma(s, t) \phi(t) d t .
$$

$X(t)$ has the expansion

$$
X(t)=\sum_{1}^{\infty} \eta_{k} \lambda_{k}^{1 / 2} \phi_{k}(t) \quad 0 \leqq t \leqq a
$$

where $\eta_{1}, \eta_{2}, \cdots$ is a sequence of independent normal mean zero variance one random variables. This expansion converges for each $t$ w.p.1. and also converges in the $L^{2}[0, a]$ mean w.p.1., i.e.,

$$
\int_{0}^{1}\left|X(t)-\sum_{1}^{n} \eta_{k} \lambda_{k}^{1 / 2} \phi_{k}(t)\right|^{2} d t \rightarrow 0 \text { as } k \rightarrow \infty .
$$

(This is proved in [4] for the case where $X(t)$ is stationary; it is well known that the results remain true in the nonstationary case.) Now each $\phi_{k}$ is continuous so using either $(\mathrm{B} 2) \Rightarrow(\mathrm{B} 1)$ or $(\mathrm{B} 3) \Rightarrow(\mathrm{B} 1)$ of Theorem $\mathrm{B}$, we have:

CoRollary 3. If $E\left\{\sup _{0 \leqq t \leqq a}|X(t)|\right\}<\infty$, the convergence in (5.1) is uniform in $t$ w.p.1.

\section{REFERENCES}

1. S. D. Chatterji, A note on the convergence of Banach space-valued martingales, Math Ann. 153 (1964), 142-149.

2. J. Delporte, Convergence uniforme presque sîre de séries de fonctions aléatoires normales presque sûrement continue, C. R. Acad. Sci. Paris 256 (1963), 3949-3952.

3. J. L. Doob, Stochastic processes, Wiley, New York, 1953.

4. M. Kac and A. J. F. Siegert, An explicit representation of a stationary gaussian process, Ann. Math. Statist. 18 (1947), 438-442.

5. R. C. Paley and N. Wiener, Fourier transforms in the complex domain, Amer. Math. Soc. Colloq. Publ., Vol. 19, Amer. Math. Soc., Providence, R. I., 1934.

6. F. S. Scalora, Abstract martingale convergence theorems, Pacific J. Math. 11 (1961), 347-374.

7. L. A. Shepp, Radon-Nikodym derivatives of gaussian measures, Ann. Math. Statist. 37 (1966), 321-354.

UNIVERSITY OF ILLINOIS 